\title{
Fertilización nitrogenada en el rendimiento de dos variedades de quinua
}

\author{
Nitrogen fertilization on yield of two varieties of quinoa
}

Eroncio Mendoza Nieto', Dionicio Luis Olivas', Cecilia Maura Mejía Domínguez¹, Judith García Cochagne ${ }^{2}$

\section{RESUMEN}

Objetivo: Establecer la curva de respuesta en el rendimiento de grano de dos variedades de quinua para las aplicaciones de dosis creciente de nitrógeno. Métodos: El ensayo se ejecutó en un suelo de $\mathrm{pH} 8,10$ y 2,60 dS/m de salinidad, usando el experimento factorial de dos variedades por cinco dos.is de nitrógeno empleando el diseño completo randomizado teniendo un total de 10 tratamientos, formados por variedades de quinua Salcedo INIA y Altiplano INIA y de factor dosis de 0, 100, 200, 300 y $400 \mathrm{~kg} / \mathrm{h}$ a de nitrógeno en forma de sulfato de amonio $(21 \% \mathrm{~N})$, a los cinco meses se realizó la evaluación de rendimiento de grano de quinua expresado en t/ha. Resultados: No se encontró diferencia estadística para el factor variedad $(\rho>0,05)$ y para la dosis de aplicación de nitrógeno, se encontró una variación significativa $(\rho \leq 0,05)$ entre los niveles del factor en estudio, al tener variaciones de valores de 1,7 a 2,8 t/ha de rendimiento para las dosis de 0 y $200 \mathrm{~kg} / \mathrm{ha}$ de nitrógeno Conclusiones: El rendimiento de quinua se incrementa hasta cierta cantidad de insumo aplicado y después disminuye debido al efecto depresivo de niveles altos de nutriente usado.

Palabras clave: Quinua, variedades, fertilización, nitrógeno, dosis.

\section{ABSTRACT}

Objective: Establish the response curve on grain yield of two varieties of quinoa for applications of increasing dose of nitrogen. Methods: The test was run on a soil pH 8.10 and $2.60 \mathrm{dS} / \mathrm{m}$ of salinity, using the factorial experiment of two varieties for five nitrogen doses using randomized complete design having a total of 10 treatments, formed by quinoa varieties Salcedo INIA and Altiplano INIA and factor doses of $0,100,200,300$ and $400 \mathrm{~kg} / \mathrm{ha}$ of nitrogen as ammonia sulfate $(21 \% \mathrm{~N})$, at five months performed the evaluation of yield of quinoa grain expressed in $t / h a$. Results: Statistical difference wasn't found for the variety factor $(\rho>0.05)$ and for nitrogen application dose, a significant variation was found $(\rho \leq 0.05)$ between the levels of the factor in study, to have variations of values from 1.7 to $2.8 \mathrm{t} / \mathrm{ha}$ of yield for doses of 0 and $200 \mathrm{~kg} / \mathrm{ha}$ of nitrogen. Conclusions: quinoa yield increases to a certain amount of input applied and then decreases due to the depressing effect of high levels of nutrient used.

Keywords: Quinoa, varieties, fertilization, nitrogen, doses.

\footnotetext{
${ }^{1}$ Universidad Nacional José Faustino Sánchez Carrión. Huacho, Perú.

${ }^{2}$ Instituto Nacional de Innovación Agraria. Lima, Perú
} 


\section{INTRODUCCIÓN}

La quinua (Chenopodium quinoa Willd.) es considerada como el único alimento vegetal que tiene todos los aminoácidos esenciales, oligoelementos y vitaminas y no contiene gluten, se califica una especie con muchas bondades nutritivas, versatibilidad agronómica, adaptabilidad desde el nivel del mar hasta 4 $000 \mathrm{msnm}$; donde otros cultivos no pueden desarrollarse, múltiples formas de uso tradicional y no tradicional, bajo costo de producción al ser un cultivo poco exigente en insumos y potencialidad para la expansión del cultivo a otros continentes para contribuir a la seguridad alimentaria y nutricional de diferentes regiones del planeta, en especial para aquellos países donde la población no tiene acceso a fuentes de proteína, donde las adversidades de suelos pobres y climas extremos no permiten promover otros cultivos (Bojanic, 2013).

Dentro de las funciones de los elementos esenciales, el nitrógeno constituye parte de las proteínas, enzimas, ácidos nucleicos y de la molécula de clorofila, por tanto es indispensable en la formación de proteínas y vital para la realización de la fotosíntesis, metabólicamente favorece el crecimiento vegetativo, mayor vigor a la planta y abundancia de hojas (Molina, 2014 y Pilarte, 2014).

Solid-OPD (2013), sostiene que los suelos de la costa son áridos, bajos en materia orgánica y nutrientes, por lo que recomienda para el cultivo de la quinua una fórmula de abonamiento de $240-200-80 \mathrm{~kg} / \mathrm{ha}$ de $\mathrm{N}-\mathrm{P}_{2} \mathrm{O}_{5}-\mathrm{K}_{2} \mathrm{O}$ aplicados en forma de urea, superfosfato triple de calcio y cloruro de potasio; además, sugiere la aplicación de fuentes de materia orgánica a la preparación del terreno sobre todo para mejorar las propiedades físicas y biológicas del suelo.

INIA (2014), menciona que en la Costa se está masificando su cultivo por su rendimientos de grano que llegan hasta 5 t/ha con las variedades de Salcedo INIA e INIA 431 Altiplano, con fórmulas de fertilización que varían de 180-240 kg/ha de nitrógeno, 80-110 $\mathrm{kg} / \mathrm{ha}$ de $\mathrm{P}_{2} \mathrm{O}_{5}$ y $100-120 \mathrm{~kg} / \mathrm{ha}$ de $\mathrm{K}_{2} \mathrm{O}$. Sin embargo, EEA-Illpa Puno (2014), sugiere una fórmula de abonamiento de 200-180-80 kg/ha de $\mathrm{N}-\mathrm{P}_{2} \mathrm{O}_{5}-\mathrm{K}_{2} \mathrm{O}$ complementado con estiércol descompuesto de entre 8 a $10 \mathrm{t} / \mathrm{ha}$. Asimismo,
Mujica, Canahua y Saravia (2014), destacan que la planta de quinua es exigente en nutrientes de nitrógeno, calcio, fósforo y potasio, por lo requiere un buen abonamiento y fertilización balanceada de $240-200-80 \mathrm{~kg} / \mathrm{ha}$ de $\mathrm{N}-\mathrm{P}_{2} \mathrm{O}_{5}-\mathrm{K}_{2} \mathrm{O}$ con aplicación de materia orgánica debido a que los suelos de la Costa son muy bajos en materia orgánica, arenosos y cantidad de nutrientes escasos.

En el año 2013, el Instituto Nacional de Innovación Agraria (INIA) inicia sus trabajos de investigación en su sede central de La MolinaLima en una parcela de observación con el propósito de impulsar la producción de quinua en nuevas zonas agrícolas del país para beneficio de los medianos y pequeños agricultores; se evaluaron ocho cultivares, de ellos Salcedo y Altiplano INIA presentaron un comportamiento agronómico mejor cuyos resultados preliminares en rendimiento fueron de 3,2 t/ha para el cultivar Salcedo INIA y de 4 t/ha con el Altiplano INIA(Andina, 2013).

Después de carbono, oxígeno e hidrógeno que la planta extrae del agua y aire, los demás elementos son absorbidos del suelo, para lo que debe existir en cantidades suficientes y en forma asimilable, el nitrógeno aplicado en cantidades mayores a las dosis estimulantes de óptima y máximo, por sus efectos depresivos conduce el descenso del rendimiento del cultivo (Urbano Terrón, 2001) y en la curva respuesta a dosis crecientes de nitrógeno, se presenta tres tramos, en el primer tramo existe respuesta a las aplicaciones de dosis crecientes, en el segundo tramo no existe respuesta y en el tercer tramo, el rendimiento disminuye al incrementarse la dosis de aplicación del nitrógeno (Oyarzun, 2010).

Después del agua, el nitrógeno es el factor limitante del crecimiento más importante para las plantas y por tanto para la producción agrícola, al influir directamente en la producción vegetal, en forma cuantitativa y cualitativamente, al favorecer el aumento de área foliar que causa el incremento de la tasa fotosintética produciendo mayor crecimiento vegetativo de los tejidos (Gonzáles, Gonzáles y Estavillo, 2003).

EI INIA reporta que en la campaña agrícola 2011-2012, las pérdidas causadas por aves plaga en el cultivo de quinua en Puno fueron del orden del $30 \%$ de la producción, ocasionando pérdidas de S/. 2172,00 por ha y pérdidas para 
la Región Puno de S/. 65892 732,86 (Delgado, 2013). Las aves perjudican la producción de quinua al consumir los granos durante las fases de fructificación y maduración y también contaminando los granos con sus excretas con lo cual dañan la calidad del producto.

El experimento se realizó con el propósito de: i) Elaborar la curva de respuesta de rendimiento de grano de quinua para las aplicaciones de dosis crecientes de nitrógeno $(\mathrm{N})$ y ii) Determinar la variedad de mayor rendimiento de grano de quinua para las condiciones edafoclimáticas de la zona de experimentación.

\section{MATERIAL Y MÉTODOS}

El ensayo se llevó a cabo en una de las parcelas agrícolas de la Universidad Nacional José Faustino Sánchez Carrión situado en el distrito de Huacho, provincia de Huaura, Región Lima, geográficamente ubicado a $11^{\circ} 5^{\prime}$ $60^{\prime \prime}$ latitud Sur, $77^{\circ} 35^{\prime} 60^{\prime \prime}$ longitud Oeste y una altitud aproximado de $30 \mathrm{msnm}$; el campo experimental tuvo las características físicas y químicas de 8,10 de $\mathrm{pH} 2,60 \mathrm{dS} / \mathrm{m}$ de salinidad, textura franco arenosa $4,3 \%$ de calcáreo total $0,76 \%$ de materia orgánica (MO), $11 \mathrm{ppm} \mathrm{P}, 180$ ppm $\mathrm{K}$ y el agua de riego se caracterizó por tener $1,80 \mathrm{dS} / \mathrm{m}$ de conductividad eléctrica 8,01 de $\mathrm{pH} 4,17 ; 1,26 ; 0,31$ y $12,54 \mathrm{meq} / \mathrm{L}$ de $\mathrm{Ca}^{2+}$, $\mathrm{Mg}^{2+}, \mathrm{K}^{+}$y $\mathrm{Na}^{+}$solubles, respectivamente y en aniones fueron de 9,$50 ; 0,00 ; 6,10 ; 0,04$ y 2,51 meq/L de los iones de $\mathrm{Cl}^{-}, \mathrm{CO}^{-}, \mathrm{HCO}_{3}^{-}, \mathrm{NO}_{3}^{-}$y $\mathrm{SO}_{4}^{-}$, teniendo el valor de la relación de adsorción de sodio (RAS) de 7,61 y clasificándose como $\mathrm{C}_{3-} \mathrm{S}_{2}$.

Se evaluaron 10 tratamientos, formados por combinación de factores de dos variedades de quinua (Altiplano INIA y Salcedo INIA) por cinco dosis de nitrógeno en razón de 0,100, 200, 300 y $400 \mathrm{~kg} / \mathrm{ha}$ de $\mathrm{N}$ en forma de sulfato de amonio $(21 \% \mathrm{~N})$ y dosis constantes de fósforo y potasio en cantidades de 160 y $200 \mathrm{~kg} / \mathrm{ha}$ de $\mathrm{P}_{2} \mathrm{O}_{5}$ y K $\mathrm{K}_{2} \mathrm{O}$ en forma de superfosfato triple de calcio ( $46 \%$ $\left.\mathrm{P}_{2} \mathrm{O}_{5}\right)$ y sulfato de potasio $\left(50 \% \mathrm{~K}_{2} \mathrm{O}\right)$.

El experimento tuvo cuatro bloques separados entre ellos por $2 \mathrm{~m}$ de espacio, en cada bloque se ubicó al azar los 10 tratamientos, y a la vez cada tratamiento estuvo ubicado en tres surcos distanciados a 0,80 m entre ellos por $3 \mathrm{~m}$ de largo, teniendo una unidad experimental o parcela (ue) de $7,20 \mathrm{~m}^{2}$. En cada surco dentro de la ue, se sembraron 300 semillas y después del desahíje quedaron 60 plantas para las evaluaciones hasta el final del ensayo. El ensayo se ejecutó empleando el experimento factorial de dos variedades de quinua por cinco dosis de nitrógeno $(0,100,200,300$ y $400 \mathrm{~kg} / \mathrm{ha}$ de $\mathrm{N}$ ) empleando el diseño bloques completo randomizado (DBCR) de 10 tratamientos con cuatro réplicas y para la comparación de los tratamientos se usó la prueba de Tukey al nivel de significación de $\alpha=0,05$.

A las tres semanas después de la siembra de quinua de las variedades de Salcedo INIA y Altiplano INIA, cuando las plántulas tenían una altura de 15 a $20 \mathrm{~cm}$, se aplicaron las dosis de 0 , $100,200,300$ y $400 \mathrm{~kg} / \mathrm{ha}$ de $\mathrm{N}$ al fondo del surco e inmediatamente se taparon con el suelo mediante el aporque y posteriormente se efectuó el riego correspondiente. Otras labores agronómicas del cultivo se realizaron de manera similar en todas las unidades experimentales durante todo el periodo vegetativo del cultivo y la evaluación de rendimiento de grano se realizó en 20 plantas del surco central, la información obtenida expresada en $\mathrm{t} \mathrm{ha}^{-1}$ se procesó empleando un programa estadístico comercial, tanto para el análisis de varianza y prueba de Tukey.

\section{RESULTADOS}

Los resultados de rendimiento de granos de quinua de las variedades Altiplano INIA y Salcedo INIA son similares al no mostrar diferencia estadística $(\rho>0,05)$ al tener valores de 2,5 y $2,3 \mathrm{t} / \mathrm{ha}$ y para la fuente de variabilidad de dosis de nitrógeno se encontró diferencia significativa $(\rho<0,05)$ al observarse los valores de rendimiento de 1,$7 ; 2,7 ; 2,8 ; 2,6$ y 2,1 t/ha para las dosis de aplicación de 0, 100, 200, 300 y $400 \mathrm{Kg} / \mathrm{ha}$ de $\mathrm{N}$ incorporado en forma de sulfato de amonio $(21 \% \mathrm{~N})$ respectivamente y para el efecto de interacción entre variedad y dosis de $\mathrm{N}$ no se encontró diferencia estadística (Tabla 1). 
Tabla 1. Análisis de varianza para rendimiento de grano de quinua (t/ha).

\begin{tabular}{llccclc}
\hline Fuente & GL & SC SeC. & SC Ajust. & CM Ajust. & F & P \\
\hline Bloque & 3 & 0,88893 & 0,88893 & 0,29631 & 3,66 & 0,025 \\
Variedad & 1 & 0,30794 & 0,30794 & 0,30794 & 3,8 & 0,062 \\
Dosis & 4 & 7,05597 & 7,05597 & 1,76399 & 21,78 & 0,000 \\
Variedad*Dosis & 4 & 0,18815 & 0,18815 & 0,04704 & 0,58 & 0,679 \\
Error & 27 & 2,18686 & 2,18686 & 0,08099 & & \\
Total & 39 & 10,62785 & & & & \\
\hline
\end{tabular}

$S=0,284596$ R-cuad. $=79,42 \%$ R-cuad. $($ ajustado $)=70,28 \%$

A pesar que en el análisis de varianza para la interacción de variedad por dosis no hubo evidencias de diferencia estadística, para la combinación de las dos variables se encontró diferencia significativa al tener respuesta de rendimiento que varían de 1,7 a 2,9 t/ha para las dosis dentro de la variedad (Tabla 2).

Tabla 2. Rendimiento promedio de grano de quinua y la prueba de Tukey a una confianza de $95 \%$

\begin{tabular}{|c|c|c|c|c|}
\hline Variedad & Dosis-N & $\mathbf{N}$ & Media & Agrupación \\
\hline Altiplano & 200 & 4 & 2,9 & A \\
\hline Altiplano & 300 & 4 & 2,8 & $A B$ \\
\hline Altiplano & 100 & 4 & 2,7 & $A B$ \\
\hline Salcedo & 200 & 4 & 2,7 & $A B C$ \\
\hline Salcedo & 100 & 4 & 2,7 & $A B C$ \\
\hline Salcedo & 300 & 4 & 2,4 & $A B C D$ \\
\hline Altiplano & 400 & 4 & 2,2 & BCD \\
\hline Salcedo & 400 & 4 & 2,0 & $C D$ \\
\hline Altiplano & 0 & 4 & 1,7 & D \\
\hline Salcedo & 0 & 4 & 1,7 & D \\
\hline
\end{tabular}

Las medias que no comparten una letra son significativamente diferentes

En la curva de respuesta de rendimiento en base a las dosis de nitrógeno para las dos variedades evaluadas (Figura 1), se observa que la variedad Altiplano INIA tuvo mejor respuesta que la variedad Salcedo INIA, para ambas variedades existe mayor respuesta hasta la dosis de aplicación de $100 \mathrm{~kg} / \mathrm{ha}$ de $\mathrm{N}$ y a partir de esta cantidad va disminuyendo la cantidad de rendimiento hasta alcanzar el máximo beneficio en la dosis de $200 \mathrm{~kg} / \mathrm{ha}$ de nitrógeno y después se reduce el rendimiento por efecto depresivo.

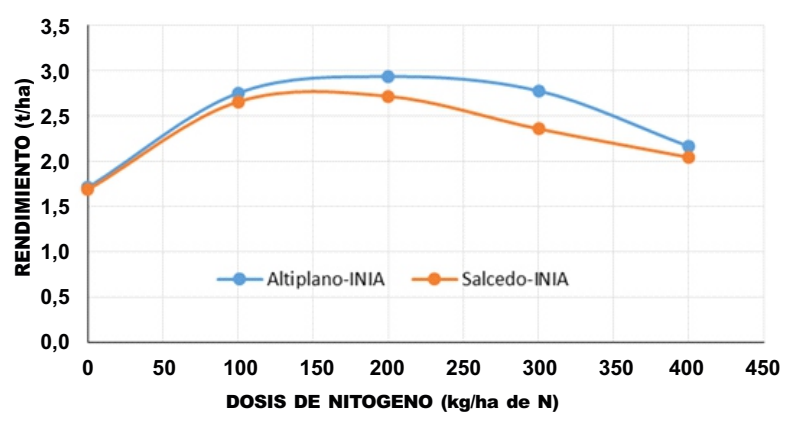

Figura 1. Efecto de dosis de nitrógeno en el rendimiento de dos variedades de quinua

\section{DISCUSIÓN}

De las dos variedades evaluadas, la variedad Altiplano INIA supera en rendimiento a la variedad Salcedo INIA al tener valores de 2,5 y 2,3 t/ha de grano de quinua para las dos variedades respectivamente, estos valores están por debajo del rendimiento potencial de 4,0 y 3,2 t/ha de grano de quinua para Altiplano y Salcedo según reporta (Andina, 2013), en ambos casos la productividad menor para las condiciones edafoclimáticas de Huacho, pueden haber sido influenciados por las causas de siembra fuera de época al realizarse a mediados de julio y por la considerable pérdida de granos causado por la presencia de aves plaga, las cuales consumieron el grano ya que el experimento se realizó en campo abierto sin ningún control químico ni físico (Delgado, 2013).

Los resultados obtenidos para la respuesta a la aplicación de dosis crecientes de nitrógeno coinciden con lo sostenido por Molina Ceballos (2014) y Pilarte Pacón (2014) quienes manifiestan que los incrementos de nitrógeno incrementan también los rendimientos pero hasta alcanzar un nivel de $200 \mathrm{~kg} \mathrm{ha}^{-1}$, y por encima de este nivel, los rendimientos disminuyen. Así también coincide con lo encontrado por Solid-OPD (2013) quienes 
sostienen que los rendimientos se incrementan al aumentar las dosis de nitrógeno, para luego alcanzar el punto óptimo y luego decaer por efecto depresivo de altas dosis de nitrógeno (Urbano, 2001).

El rendimiento del grano de quinua aumenta con el incremento de dosis de aplicación hasta una dosis de $100 \mathrm{~kg} / \mathrm{ha}$ de $\mathrm{N}$, después el incremento de rendimiento disminuye hasta llegar a un máximo a una dosis de $200 \mathrm{~kg} / \mathrm{ha}$ y después disminuye la cantidad cosechada ajustándose la curva a la ley de rendimientos decrecientes (Oyarzun, 2010) y el efecto depresivo que causa la aplicación de altas dosis del nitrógeno (Urbano, 2001).

\section{REFERENCIAS BIBLIOGRÁFICAS}

Andina. Agencia Peruana de Noticias. (Enero, 2013). Quinua podría cultivarse en la costa central y valles de Arequipa: INIA. $\mathrm{R}$ e c u p e r a d o d e http://www.andina.com.pe/agencia/notic ia-quinua-podria-cultivarse-la-costacentral-y-valles-arequipa-inia444872.aspx

Bojanic, A. (2011). La quínua: cultivo milenario para contribuir a la seguridad alimentaria mundial. Retrieved Octubre, 18, 2014.

Delgado, P. (2013). Aves plaga del cultivo de quinua (Chenopodium quinoa Willd.) y alternativas de control". Contrato de subvención $N^{\circ}$ 343-2012-INIACONCYTEC-OAJ. Recuperado de: http://portal.concytec.gob.pe/images/sto ries/images2013/agosto/quinua/present acion_pedro_delgado_mamani.pdf

EEA Illpa-Puno. (2014). Quinua Salcedo INIA. Tríptico producido por la Estación Experimental Agraria Illpa - Puno del Instituto Nacional de Innovación Agraria - INIA.

Gonzáles, M.C., Gonzáles, M.B. \& Estavillo, A. J. Ma. (2004). Capítulo 13 Nitrógeno, Agricultura y Medio Ambiente. En Thomson Editores Spain, Ecofisiología Vegetal - Una ciencia síntesis (pp. 387 411).
INIA. (2014). Manejo integrado del cultivo de quinua en la costa. Dirección de Investigación Agraria - Instituto Nacional de Innovación Agraria - INIA La Molina. Hoja divulgativa $\mathrm{N}^{\circ} 3-2014$.

Molina Ceballos, Javier A. (2014). El nitrógeno y Fertilizantes nitrogenados. Recuperado de

http://www.monografias.com/trabajos82 I n itroge no-fertilizantes nitrogenados/nitrogeno-fertilizantesnitrogenados.shtml

Mujica Angel, Canahua Alipio \& Saravia Raúl. (2014). Agronomía del cultivo de la quinua - Abonamiento y fertilización. Recuperado de: http://www.rlc.fao.org/es/agricultura/pro du/cdrom/contenido/libro03/cap2.htm.

Oyarzum, M. 2010. Respuesta productiva de un cultivo de maíz (Zea mays Dracma) a distintas dosis de nitrógeno con dos tipos de riego (Aspersión e inundación) y efecto sobre la lixiviación de nitratos. Tesis. Universidad Pública de Navarra. Navarra, España.

Pilarte Pavón, Felipe. (2014). Función de los elementos esenciales en los cultivos $\mathrm{N}$ i t r óg e no. R e c u p e r a d o de:http://www.a4n.com.sv/uploaded/mo d documentos/Funci_n\%20de\%20los $\% 20$ elementos $\% 20$ esenciales $\% 20$ en $\%$ 2010 \%20cultivos.pdf.

Solid OPD. (Noviembre, 2013). Modulo I: Tecnología productiva de la quinua. Organización Privada de Desarrollo O P D. R e c u p e r a d o d e : http://www.solidinternational.ch/wpcontent/themes/solid/sources/img/Quin ua-Marco-Referencial1.pdf

Urbano Terrón, Pedro. (2001). Tratado de Fitotecnia General. 2da Edición. Madrid: Ediciones Mundi-prensa.

\section{Correo electrónico:}

emendozan@unjfsc.edu.pe

Revisión de pares:

Recibido: 25-04-2016

Aceptado: 24-06-2016 